\title{
Cytokine Effect of TLR3, TLR4, and TLR7 Agonists Alone or Associated with Leishmania infantum Antigen on Blood from Dogs
}

\author{
Pamela Martínez-Orellana, Sara Montserrat-Sangrà, Paulina Quirola-Amores, \\ Noemí González, and Laia Solano-Gallego \\ Departament de Medicina i Cirurgia Animals, Facultat de Veterinària, Universitat Autònoma de Barcelona, Bellaterra, Spain \\ Correspondence should be addressed to Laia Solano-Gallego; laia.solano@uab.cat
}

Received 13 July 2018; Revised 30 September 2018; Accepted 30 October 2018; Published 12 November 2018

Academic Editor: Roberto Amerigo Papini

Copyright (C) 2018 Pamela Martínez-Orellana et al. This is an open access article distributed under the Creative Commons Attribution License, which permits unrestricted use, distribution, and reproduction in any medium, provided the original work is properly cited.

\begin{abstract}
Activation of toll-like receptors (TLRs) has been shown to play an important role in leishmaniosis by enhancing the parasite specific immune responses to control infection. However, the role of TLR agonists has not been studied in detail in dogs. The aim of this study was to determine the effect of TLR3, TLR4, and TLR7 agonists (TLR3a, TLR4a, and TLR7a) alone or in combination with Leishmania infantum antigen (LSA) on TNF- $\alpha$ and IL-6 production in blood from dogs living in endemic areas of canine leishmaniosis (CanL). Twenty-four healthy dogs from Catalonia $(n=14)$ and Ibizan hound dogs from the island of Mallorca ( $n=10)$ were enrolled. Whole blood with TLR3a, TLR4a, and TLR7a alone or combined with LSA were cultured separately, and IFN- $\gamma$, TNF- $\alpha$, and IL- 6 were measured by ELISA. A significant increase of TNF- $\alpha$ was found for all conditions studied compared to medium alone. Stimulation with TLR4a $(p=0.0001)$ and TLR7a $(p=0.005)$ presented a significantly marked increase in TNF- $\alpha$ and IL-6 production compared to TLR3a. Importantly, significantly higher TNF- $\alpha$ production was found in LSA+TLR4a ( $p=0.0001)$ stimulated blood and LSA+TLR7a ( $p=0.005)$ compared to LSA alone. All dogs showed higher TNF- $\alpha$ production after LSA+TLR7a compared to TLR7a $(p=0.047)$ and LSA+TLR3a compared to TLR3a $(p=0.052)$. These data indicate a marked inflammatory cytokine effect of TLR4a and TLR7a on blood from healthy dogs living in endemic areas of CanL. Additionally, LSA+TLR7a promoted a synergistic proinflammatory effect with TNF- $\alpha$ in all dogs. Those findings suggest an active role of TLRs in proinflammatory responses, which might be strongly involved in the process of disease resolution.
\end{abstract}

\section{Introduction}

Leishmaniosis are a group of protozoan diseases caused by several species of Leishmania (class Kinetoplastea, family Trypanosomatidae). More than 70 countries are endemic for Leishmania infection, including countries in the Mediterranean basin, the Middle East, Asia, Africa, and South America [1]. Leishmania infantum is the species most commonly associated with canine infections [2]. In endemic Mediterranean areas, dogs are infected through the bite of sand flies of the genus Phlebotomus in the warmer months, from April to November [3-5].

Canine leishmaniosis (CanL) can be manifested with a range of clinical signs and laboratory abnormalities that include nonpruritic skin lesions such as exfoliative dermatitis and ulcerations, local or generalized lymphadenomegaly, weight loss, poor appetite, ocular lesions, epistaxis, lameness, renal failure, and diarrhea [6]. In addition, different degrees of disease severity exist [6]. Moreover, persistent subclinical infections in dogs are common in endemic areas such as the Mediterranean basin [7, 8].

Dogs of any breed are susceptible to L. infantum infection, but breeds such as Bulldogs, Dobermanns [9], Boxers, German Shepherds, and Rottweilers seem to be more susceptible to develop CanL [2]. However, Ibizan hounds are especially CanL-resistant to develop the disease living in endemic areas such as the island of Mallorca [10]. Mixed T helper 1 and T helper 2 (Th1/Th2) responses with a dominant Th1 profile are required by humans and dogs for protection against viscerotropic infection by $L$. donovani and $L$. infantum [11, 
12]. Classically, Th1 responses are associated with resistance and Th2 responses are associated with susceptibility in dogs $[13,14]$, humans [15], and rodent models [16]. Moreover, it is demonstrated in both canine and murine models that Th1 cytokines as IFN- $\gamma$ and TNF- $\alpha$ activate macrophages to kill L. infantum via increasing the nitric oxide (NO) pathway [1719].

The cell-mediated immune (CMI) response is the major defence against CanL $[20,21]$ and is indispensable for the resolution of this parasitic infection $[22,23]$. One clear example is observed in Th1 responses developed by CanL-resistance Ibizan hound [10]. In contrast, disease development and severity in CanL is often correlated with a marked humoral response and the abrogation of Th1 cytokines production $[24,25]$, which cannot control the infection.

Toll-like receptors (TLRs) are type 1 membrane proteins that belong to the group of patter recognition receptors (PRRs) [26]. Ten TLRs are found in dogs [27] and they bind conserved molecular structures found in large groups of pathogen-associated molecular patterns (PAMPs). TLRs are located in either plasma membrane or internal membranes mainly of macrophages, dendritic cells (DC), natural killer (NK) cells, and lymphocytes (T and B) and induce proinflammatory cytokines, type-1 IFN, chemokines, and costimulatory molecules $[26,28]$ as well as shaping adaptive immunity [29]. TLRs have been shown to play an important role in leishmaniosis [30] because they are one of the most important nonclonal sets of PRRs families [28].

There are several studies that have demonstrated the importance of TLR3 [31, 32], TLR4 [33], and TLR7 [34] in recognition, control, and protection against Leishmania infection. Those previous works are based mainly on investigations of Leishmania major in a murine model [30]. However, limited information is available about the function of TLRs in canine L. infantum infection [20].

Toll-like receptor agonists (TLRa) are natural and synthetic molecular structures (PAMPs)[35] that bind to TLRs with powerful immunostimulant potential. The ability of TLRa is to activate signalling pathways to manage innate and acquired immunity [36]. Therefore, they amplify parasite immune responses by stimulating the production of proinflammatory cytokines, which might play an important role in controlling Leishmania infection [37]. The current treatment of CanL is not sufficiently effective; thus the use TLR ligands as adjuvants for vaccine or as immunotherapy could be an interesting tool against CanL. Previously, TLR2 agonist [Pam3CSK4, a synthetic derivative of triacylated lipoproteins (TLR2a)] used alone was shown to enhance the production of the proinflammatory cytokines like TNF- $\alpha$ and IL-6 in dogs with clinical leishmaniosis, infected "resistant" and healthy noninfected. Furthermore, a combination of $L$. infantum soluble antigen (LSA)+TLR2a promoted a synergistic proinflammatory effect with TNF- $\alpha$ in Ibizan hounds [38]. The hypothesis of this study was that TLR3, TLR4, and TLR7 agonists (TLR3a, TLR4a, and TLR7a) alone will enhance the production of proinflammatory cytokines in canine ex vivo whole blood. In addition, we hypothesized that the combination of TLRa with LSA promotes a synergistic release of proinflammatory cytokines when compared with
LSA or TLR ligands alone. Therefore, the main objective of the present study was to investigate and expand the knowledge on the effect of others TLRa different from TLR2a as TLR3a [Poly (I:C)], TLR4a [Monophosphoryl lipid A (MPLA)], and TLR7a [Imiquimod-R837 (IMQ)] alone or in combination with L. infantum antigen on ex vivo whole blood from apparently healthy dogs living in an endemic area of leishmaniosis (Catalonia and the island of Mallorca, Spain).

\section{Materials and Methods}

2.1. Dogs and Sampling. The study was conducted in Camprodon (Catalonia, Spain) in July $2015(n=14)$ and island of Mallorca (Balearic Islands, Spain) in February $2017(\mathrm{n}=10)$, both endemic areas of CanL. Twenty-four apparently clinically healthy outdoors dogs of both sexes (eleven females and thirteen males) were enrolled. Age ranged between 8 month and 9 years and the median was 3.5 years old. Veterinarians subjected all dogs to physical examination and found that they did not present any clinical signs and were apparently healthy with the exception of three dogs that presented only papular dermatitis due to L. infantum, a mild form of clinical leishmaniosis with commonly good prognosis in dogs [39].

Collection of blood samples for this study was performed in accordance with veterinary protocols under aseptic conditions. Approximately 7-10 mL of blood from jugular or cephalic venipuncture was collected and immediately transferred into sterile tubes divided into: 1 tube with sodium heparin ( $6 \mathrm{~mL}$ volume) for whole blood assay, 1 tube with ethylenediaminetetraacetic acid (EDTA) (2mL volume), and 1 tube for serum with clotting accelerator (2-4 $\mathrm{mL}$ volume) for L. infantum specific antibodies and hematology profile. Blood sampling was obtained as screening for L. infantum infection and for checking general health status.

The dog owners gave their consent for blood sampling. Detection of antibodies against $L$. infantum using an in-house quantitative enzyme linked immunosorbent assay (ELISA) was performed on sera of all dogs as previously described and blood Leishmania real-time polymerase chain reaction (RT-PCR) was performed in 21 out of 24 dogs as previously described [40].

2.2. Whole Blood Cytokine Release Assay. Heparinized whole blood cytokine release assay was performed as previously described [38]. Briefly, blood from Mallorca dogs $(n=10)$ was stimulated as follows: (1) medium alone $(\phi),(2)$ medium with L. infantum soluble antigen (LSA) (provided by Dr. Cristina Riera, Facultat de Farmacia, Universitat de Barcelona) at a working concentration of $10 \mu \mathrm{g} / \mathrm{mL}(\phi+\mathrm{LSA})$, (3) medium with concanavalin A (ConA) a strong lymphocyte mitogen (Medicago ${ }^{\circledR}$ Uppsala, Sweden) at a working concentration of $10 \mu \mathrm{g} / \mathrm{mL}$ ( $\phi+$ ConA), (4) medium with TLR3a [Poly(I:C) Invivogen ${ }^{\circledR}$ San Diego, USA] at a working concentration of $10 \mu \mathrm{g} / \mathrm{mL}(\phi$ TLR3a), or TLR4a [Monophosphoryl lipid A (MPLA), Invivogen ${ }^{\circledR}$ San Diego, USA] at a working concentration of $1 \mu \mathrm{g} / \mathrm{mL}$ ( $\phi$ TLR4a), or TLR7a [ImiquimodR837 (IMQ) Invivogen ${ }^{\circledR}$, San Diego, USA] at a working concentration of $5 \mu \mathrm{g} / \mathrm{mL}$ ( $\phi$ TLR7a) and (5) medium with TLR3a, TLR4a or TLR7a at concentrations described above 
and LSA at a concentration described above (LSA+TLR3a or TLR4a or TLR7a). In the case of Catalonian dogs $(n=14)$, blood was stimulated with all TLRa with the exception of TLR7a. All conditions were run in duplicate and incubated for 48 hours $(\mathrm{h})$ and 5 days at $37^{\circ} \mathrm{C}$ in $5 \% \mathrm{CO}_{2}$ enriched environment. After $48 \mathrm{~h}$ and 5 days the samples were collected and centrifuged individually at $300 \mathrm{~g}$ for 10 minutes and the supernatants were collected and stored at $-80^{\circ} \mathrm{C}$ until used. Cytokine concentrations were measured in supernatants collected at $48 \mathrm{~h}$ for IL- 6 and TNF- $\alpha$ and at 5 days for IFN$\gamma$. Supernatants collected at 5 days for IFN $-\gamma$ were only stimulated with medium, LSA and ConA.

2.3. Sandwich ELISA for Canine Cytokines. Cytokine analysis of IFN- $\gamma$, TNF- $\alpha$, and IL- 6 was performed according to manufacturer's instructions (DuoSet ${ }^{\circledR}$ ELISA by Development System R\&D TM, Abingdon, UK) using 96-well plate flat bottom (Costar ${ }^{\circledR}$ Corning, New York, USA). Slight modifications were done for IFN- $\gamma$ ELISA $[24,25]$. Standard concentrations of IFN- $\gamma$ started with $2000 \mathrm{pg} / \mathrm{mL}$ followed by twofold dilutions in reagent diluent (R\&D systems, Minneapolis, USA) until $31.25 \mathrm{pg} / \mathrm{mL}$ was reached. Standard curve for TNF- $\alpha$ started with $1000 \mathrm{pg} / \mathrm{mL}$ and twofold dilutions were made until $15.6 \mathrm{pg} / \mathrm{mL}$ concentration. Standard curve for IL-6 started with $4000 \mathrm{pg} / \mathrm{mL}$ and twofold dilutions were made until $62.5 \mathrm{pg} / \mathrm{mL}$ concentration. All values under the last standard concentration were considered undetectable for each cytokine [24]. Duplicates of all supernatants studied were performed in all ELISAs. Optical density was measured with an ELISA reader (Anthos Reader 2020, Cambridge, UK) at wavelength of $450 \mathrm{~nm}$. The standard curve for each cytokine was calculated using a computer generated four parameter logistic curve-fit with program myassays (http://www.myassays.com/). Plate was repeated when the R2-value of standard curve was below 0.98. Dogs were classified as IFN- $\gamma$ producers when $L$. infantum specific IFN$\gamma$ concentration was detectable after subtracting medium alone. Dogs were classified as IFN- $\gamma$ non-producers when $L$. infantum specific IFN- $\gamma$ concentration subtracting medium alone was at undetectable levels [25].

2.4. Statistical Analysis. A nonparametric Wilcoxon signed rank test was used to compare among related several treatments. Differences were considered significant with a $5 \%$ significance level $(p<0.05)$. The statistical analysis was performed using the SPSS 17.0 for Windows software (SPSS Inc., USA). Graph was performed using excel GraphPad Prism 7 (GraphPad Software, La Jolla, USA).

\section{Results}

3.1. Clinical Data. All 14 dogs from Catalonia were clinically healthy based on physical examination and hematological parameters. Furthermore, all dogs were seronegative and only four out of 14 dogs from Catalonia were low positive to RTPCR (Table 1). There were nine males and five females with a median of age of 50.5 months and an age range from 12 months to 9 years. Four mixed Fox hounds, two Dachshunds,

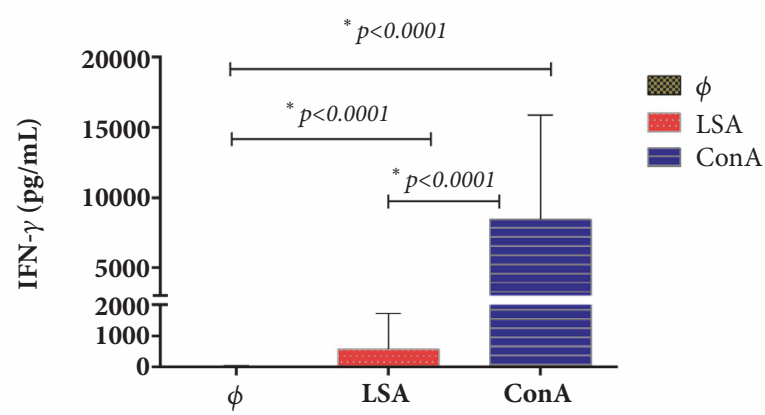

FIGURE 1: LSA specific IFN- $\gamma$ production in blood ex vivo after LSA and ConA stimulation in all dogs studied. $\Phi$ : medium alone; LSA: Leishmania soluble antigen; ConA: concanavalin A; IFN- $\gamma$ : Interferon-gamma.

two Griffons nivernais, one mixed English pointer, two Arieges pointers, one Ibizan hound, one English setter, and one Bruno Jura hound were studied.

Seven out of 10 dogs from island of Mallorca were seronegative for L. infantum antibodies and three were low positive, two of them with papular dermatitis and one without any lesion. However, all dogs tested $(n=7)$ resulted negative to blood RT-PCR. The remaining three dogs were not tested due to lack of sample. There were six females and four males. All ten dogs were Ibizan hounds. The median of age was 30 months with a range from 8 months to 6 years.

3.2. Specific L. infantum Antibody Levels, Blood RT-PCR, and IFN- $\gamma$ Production. The results from specific $L$. infantum antibody levels, IFN- $\gamma$ production, and blood RT-PCR from each individual dog tested $(\mathrm{n}=24)$ are summarized in Table 1 .

Clinically healthy dogs $(n=24)$ presented a mean \pm standard deviation (mean \pm SD): $16.6 \pm 21.6$ ELISA units (EU) of specific $L$. infantum antibody levels.

Levels of IFN- $\gamma$ of all animals under study $(\mathrm{n}=24)$ after LSA stimulation (mean \pm SD of $584.5 \pm 1131.4 \mathrm{EU}$ ) and ConA stimulation (mean \pm SD of $8433.2 \pm 7457.1 \mathrm{EU}$ ) were significantly higher than medium alone (mean \pm SD of 12.0 $\pm 29.3 \mathrm{EU})$. The results from IFN- $\gamma$ production at 5 days after stimulation with LSA and ConA from all dogs studied are shown in Figure 1. Additionally, fourteen (58.3\%) out of twenty-four dogs were classified as parasite-specific IFN- $\gamma$ producers (Table 1).

3.3. TNF- $\alpha$ Concentration. The results of TNF- $\alpha$ concentration for each treatment condition in all dogs studied $(n=24)$ are summarized in Table 2 . There was a significant increase of TNF- $\alpha$ production in samples supplemented with TLR4a $(p=0.0001)$ and TLR7a $(p=0.005)$ when compared with TLR3a in all dogs studied and also on TLR4a $(p=0.0001)$ and TLR7a $(p=0.005)$ in combination with LSA when compared with LSA+TLR3a. Concentration of TNF- $\alpha$ of all animals studied was significantly higher for all treatments $[\phi+\operatorname{LSA}(p=0.005), \phi+\operatorname{ConA}(p=0.0001)$, $\phi+$ TLR3a $\quad(p=0.044), \quad \phi+$ TLR4a $\quad(p=0.0001), \quad \phi+$ TLR7a 
TABLE 1: Clinical, serological, molecular, and parasite specific IFN- $\gamma$ production from each individual dog tested $(\mathrm{n}=24)$.

\begin{tabular}{|c|c|c|c|c|c|c|c|c|c|}
\hline \multirow{2}{*}{$\begin{array}{l}\mathbf{N}^{\circ} \\
1\end{array}$} & $\begin{array}{c}\text { SGE } \\
\text { (MONTHS) }\end{array}$ & $\begin{array}{l}\text { GNALMENT } \\
\text { BREED }\end{array}$ & SEX & \multicolumn{2}{|c|}{$\begin{array}{l}\text { LSA IFN- } \boldsymbol{\gamma}(\mathrm{pg} / \mathrm{mL}) \\
(\text { INTERPRETATION) }\end{array}$} & \multicolumn{2}{|c|}{$\begin{array}{c}\text { ELISA UNITS } \\
\text { (INTERPRETATION) }\end{array}$} & \multicolumn{2}{|c|}{$\begin{array}{c}\text { RT-PCR } \\
\text { (parasite/mL) } \\
\text { (INTERPRETATION) }\end{array}$} \\
\hline & 96.0 & $\begin{array}{l}\text { FOX HOUND } \\
\text { mixed }\end{array}$ & $\mathrm{M}$ & 0.0 & (NP) & 5.2 & $(-)$ & 0.5 & $(+)$ \\
\hline 2 & 24.0 & $\begin{array}{l}\text { FOX HOUND } \\
\text { mixed }\end{array}$ & F & 993.6 & $(\mathrm{P})$ & 4.4 & $(-)$ & 0.0 & $(-)$ \\
\hline 3 & 60.0 & $\begin{array}{l}\text { ARIEGE } \\
\text { POINTER }\end{array}$ & $\mathrm{F}$ & 10.7 & (NP) & 4.1 & $(-)$ & 0.0 & $(-)$ \\
\hline 4 & 24.0 & $\begin{array}{l}\text { FOX HOUND } \\
\text { mixed }\end{array}$ & $\mathrm{M}$ & 0.0 & (NP) & 2.9 & $(-)$ & 0.0 & $(-)$ \\
\hline 5 & 60.0 & $\begin{array}{l}\text { FOX HOUND } \\
\text { mixed }\end{array}$ & M & 41.5 & (P) & 4.2 & $(-)$ & 0.0 & $(-)$ \\
\hline 6 & 72.0 & $\begin{array}{l}\text { GRIFFON } \\
\text { NIVERNAIS }\end{array}$ & M & 95.5 & (P) & 7.6 & $(-)$ & 0.0 & $(-)$ \\
\hline 7 & 12.0 & $\begin{array}{l}\text { GRIFFON } \\
\text { NIVERNAIS }\end{array}$ & M & 314.2 & (P) & 7.9 & $(-)$ & 0.5 & $(+)$ \\
\hline 8 & 48.0 & $\begin{array}{l}\text { ARIEGE } \\
\text { POINTER }\end{array}$ & M & 0.0 & (NP) & 6.1 & $(-)$ & 0.2 & $(+)$ \\
\hline 9 & 72.0 & $\begin{array}{l}\text { IBIZAN } \\
\text { HOUNDS }\end{array}$ & $\mathrm{M}$ & 920.4 & (P) & 3.0 & $(-)$ & 0.0 & $(-)$ \\
\hline 10 & 12.0 & $\begin{array}{l}\text { BRUNO DE } \\
\text { JURA }\end{array}$ & F & 0.0 & (NP) & 3.7 & $(-)$ & 0.0 & $(-)$ \\
\hline 11 & 108.0 & $\begin{array}{l}\text { ENGLISH } \\
\text { POINTER } \\
\text { mixed }\end{array}$ & F & 0.0 & (NP) & 10.1 & $(-)$ & 0.0 & $(-)$ \\
\hline 12 & 48.0 & DACHSHUNDS & M & 10.0 & $(\mathrm{NP})$ & 4.0 & $(-)$ & 0.0 & $(-)$ \\
\hline 13 & 36.0 & DACHSHUNDS & M & 285.1 & (P) & 5.1 & $(-)$ & 0.0 & $(-)$ \\
\hline 14 & 36.0 & $\begin{array}{l}\text { ENGLISH } \\
\text { SETTER }\end{array}$ & F & 142.9 & $(\mathrm{P})$ & 4.9 & $(-)$ & 0.7 & $(+)$ \\
\hline 15 & 48.0 & $\begin{array}{l}\text { IBIZAN } \\
\text { HOUNDS }\end{array}$ & M & 1635.0 & $(\mathrm{P})$ & 81.4 & $(+)$ & 0.0 & $(-)$ \\
\hline 16 & 36.0 & $\begin{array}{l}\text { IBIZAN } \\
\text { HOUNDS }\end{array}$ & M & 4663.0 & (P) & 34.5 & $(-)$ & 0.0 & $(-)$ \\
\hline 17 & 24.0 & $\begin{array}{l}\text { IBIZAN } \\
\text { HOUNDS }\end{array}$ & M & 3196.0 & (P) & 16.6 & $(-)$ & 0.0 & $(-)$ \\
\hline $18 *$ & 8.0 & $\begin{array}{l}\text { IBIZAN } \\
\text { HOUNDS }\end{array}$ & F & 619.5 & (P) & 78.9 & $(+)$ & 0.0 & $(-)$ \\
\hline $19 *$ & 8.0 & $\begin{array}{l}\text { IBIZAN } \\
\text { HOUNDS }\end{array}$ & $\mathrm{F}$ & 14.8 & $(\mathrm{NP})$ & 19.3 & $(-)$ & 0.0 & $(-)$ \\
\hline $20 *$ & 8.0 & $\begin{array}{l}\text { IBIZAN } \\
\text { HOUNDS }\end{array}$ & $\mathrm{F}$ & 0.0 & (NP) & 37.5 & $(+)$ & 0.0 & $(-)$ \\
\hline 21 & 24.0 & $\begin{array}{l}\text { IBIZAN } \\
\text { HOUNDS }\end{array}$ & $\mathrm{F}$ & 0.0 & $(\mathrm{NP})$ & 10.8 & $(-)$ & 0.0 & $(-)$ \\
\hline 22 & 24.0 & $\begin{array}{l}\text { IBIZAN } \\
\text { HOUNDS }\end{array}$ & $\mathrm{F}$ & 119.7 & (P) & 21.3 & $(-)$ & & \\
\hline 23 & 72.0 & $\begin{array}{l}\text { IBIZAN } \\
\text { HOUNDS }\end{array}$ & $\mathrm{F}$ & 574.5 & (P) & 10.6 & $(-)$ & & \\
\hline 24 & 48.0 & $\begin{array}{l}\text { IBIZAN } \\
\text { HOUNDS }\end{array}$ & $\mathrm{M}$ & 165.9 & (P) & 16.8 & $(-)$ & & \\
\hline
\end{tabular}

* Dogs that presented stage I papular dermatitis.

LSA: Leishmania soluble antigen, IFN- $\gamma$ : Interferon-gamma, pg: picograms, mL: milliliter, ELISA: enzyme linked immunosorbent assay, RT-PCR: real time polymerase chain reaction, M: male, F: female, NP: non-IFN- $\gamma$ producer, P: IFN- $\gamma$ producer, (-): negative, (+): positive, and N/D: no data. 
TABLE 2: Results of TNF- $\alpha$ and IL-6 concentrations after stimulation at each condition in all dogs studied ( $\mathrm{n}=24)$.

\begin{tabular}{|c|c|c|c|c|c|c|c|c|c|}
\hline \multirow{2}{*}{ Cytokines (pg/mL) } & \multicolumn{8}{|c|}{ Treatment conditions $($ mean \pm SD) $*$} & \multirow[b]{2}{*}{ LSA+TLR7a } \\
\hline & $\Phi$ & $\phi+$ LSA & $\phi+\operatorname{Con} A$ & $\phi+$ TLR3a & $\phi+$ TLR4a & $\phi+$ TLR7a & LSA+TLR3a & LSA+TLR4a & \\
\hline TNF- $\alpha$ & $9.6 \pm 17.1$ & $27.1 \pm 32.7$ & $107.7 \pm 107.7$ & $17.2 \pm 28.0$ & $116.3 \pm 10.9$ & $92.0 \pm 56.1$ & $18.8 \pm 17.2$ & $116.1 \pm 73.8$ & $157.1 \pm 78.1$ \\
\hline IL-6 & $15.1 \pm 19.5$ & $12.6 \pm 19.0$ & $24.3 \pm 22.7$ & $17.0 \pm 26.8$ & $76.7 \pm 39.9$ & $55.1 \pm 32.2$ & $16.9 \pm 20.4$ & $80.6 \pm 39.3$ & $75.4 \pm 39.7$ \\
\hline
\end{tabular}

* Mean \pm standard deviation (mean \pm SD), medium alone $(\Phi)$; Leishmania soluble antigen (LSA); Concanavalin A (ConA); TLR3 [Poly(I:C)] agonist ( $\Phi+$ TLR3a) and TLR3a and LSA (LSA+ TLR3a); TLR4 (MPLA) agonist ( + +TLR4a) and TLR4a and LSA (LSA+ TLR4a); TLR7 (IMQ) agonist ( + +TLR7a) and TLR7a and LSA (LSA+ TLR7a)

$(p=0.005), L S A+T L R 3 a(p=0.025), L S A+T L R 4 a(p=0.0001)$, and LSA+TLR7a $(p=0.005)]$ when compared to medium alone. Also, $\phi+$ TLR4a $(p=0.0001)$ and $\phi+$ TLR7a $(p=0.013)$ highly stimulated the production of TNF- $\alpha$ when compared to LSA alone. Moreover, higher production of TNF- $\alpha$ was observed after stimulation with LSA+TLR4a $(p=0.0001)$ and LSA+TLR7a $(p=0.005)$ compared to LSA alone. Also, after LSA+TLR3a stimulation levels of TNF- $\alpha$ were higher than $\phi+$ TLR3a stimulation $(p=0.052)$.

3.4. IL-6 Concentration. The results of IL- 6 concentration for each condition in all dogs studied $(n=24)$ are summarized in Table 2. As observed with TNF- $\alpha$, a significant increase of IL- 6 production in TLR4a $(p=0.001)$ and TLR7a $(p=0.005)$ stimulated blood was found when compared with TLR3a in all dogs studied and also in TLR4a $(p=0.001)$ and TLR7a $(p=0.005)$ stimulated blood in combination with LSA compared with LSA+TLR3a. Concentration of IL-6 of all animals studied was significantly higher after $\phi+$ ConA $(p=0.019)$, $\phi+$ TLR4a $(p=0.0001), \phi+$ TLR7a $(p=0.008), \quad$ LSA+TLR4a $(p=0.0001)$, and LSA+TLR7a $(p=0.005)$ treatments when compared to medium alone. Also, $\phi+$ TLR4a $(p=0.0001)$ and $\phi+$ TLR7a ( $p=0.007$ ) highly stimulated the production of IL- 6 when compared to LSA alone. Moreover, higher production of IL-6 was observed after LSA+TLR4a $(p=0.0001)$ and LSA+TLR7a $(p=0.005)$ compared to LSA alone.

\section{Discussion}

The present results indicated for the first time a pronounced effect of TLR4a and TLR7a on cytokine production in blood from apparently healthy dogs living in endemic areas of CanL suggesting an active role of the innate and proinflammatory immune responses induced by these TLRa when compared with other TLRa or treatment conditions. In contrast, stimulation of TLR3a seems to have a less marked effect on cytokine production, supported by a statistically significant smaller production of proinflammatory cytokines when compared with TLR4a and TLR7a.

Improvement of vaccines and treatment strategies is imperative against leishmaniosis. In this context, new adjuvants are required to elicit an intense immune response needed for protection [41]. In particular, the vaccine adjuvant properties of imiquimod (TLR7a) and resiquimod (TLR7/8a) have been previously studied $[42,43]$. Interestingly, very promising results were obtained from dogs stimulated blood with a synergic proinflammatory effect of TLR7a when combined with LSA, reflected in a higher production of
TNF- $\alpha$. A similar synergic effect of TLR2a in Ibizan hounds was previously observed but it was slightly less intense that the one observed here for TLR7a [38].

In agreement with the effect of TLR7a associated with LSA in this study, a previous L. major mouse model study described TLR7a and/or TLR7/8a as adjuvants [44]. It was found that Leishmania antigen alone was not protective to subsequent challenge unless administrated with TLR7/8a [44]. According to the synergic effect observed in the present study, Emami et al. detected an inferior Th1 response induced in Balb/c mice by TLR7a nanoliposomal immunization alone when compared to LSA plus TLR7a formulations, suggesting that TLR7a did not function well in the absence of antigen [45]. Contrarily, another study in a murine model showed that TLR7/8a seems to decrease/inhibit cytokine production induced by LSA [46]. Therefore, TLR7a appear to be good candidates for use as adjuvants for vaccines and immunotherapy in dogs. On the other hand, several studies performed in murine models and in humans showed promising results for the use of TLR7a as treatment in cutaneous [47] and visceral leishmaniasis [34]. Further studies are needed in order to characterize the therapeutic role of TLR7a in CanL.

Ligands of TLR4 have been broadly used in human vaccines as adjuvants and are the only TLRa approved due to their safety and the potent enhancement of the immune response [48]. In the present study, a marked cytokine production was observed after blood stimulation with a TLR4a alone and also when associated with LSA, supporting the role of TLR4 ligands as adjuvant. Recently, several studies have evaluated the use of TLR4a as adjuvant during Leishmania infection. Particularly, a vaccine antigen formulated with the TLR4a Glucopyranosyl lipid adjuvant in stable emulsion (GLA-SE) proved to induce antigen-specific responses that protected mice against Leishmania donovani infection and also demonstrated that prophylactic mouse immunization resulted in a marked reduction of the parasite spleen burden together with induction of memory CD4+ T lymphocytes enhancing IFN- $\gamma$, TNF- $\alpha$, and IL-2 production [49]. Moreover, an innovative virus-like particles (VLP) vaccine loaded with sand fly saliva antigen plus Leishmania antigens and GLA-SE as adjuvant improved both cellular and humoral immune responses in a murine model and also in human peripheral blood mononuclear cells (PBMCs) [50].

In agreement with the results presented here, a previous study revealed a significant downregulation of several TLRs including TLR4 during disease progression in lymph nodes of experimentally infected dogs [51] suggesting a role of TLR4 in controlling infection and protect animals as shown in murine 
models of L. major infection $[33,52]$. In contrast, so far, TLR4 transcription in unstimulated blood appears to be similar in healthy dogs and in dogs with different clinical stages of CanL at the time of diagnosis as well as during treatment follow-up $[53,54]$. Further studies are warranted to elucidate the role of TLR4 in dogs in different clinical conditions and tissues.

The higher production of TNF- $\alpha$ and IL- 6 after TLR4a blood stimulation when compared to untreated blood is in agreement with several studies carried out in murine and human leishmaniasis where TLR4 regulated the initial proinflammatory response $[33,55]$. Additionally, and as observed by others [56], stimulation with LSA+TLR4a showed an augmentation in TNF- $\alpha$ production when compared with LSA stimulus alone. Particularly, a similar study to the one presented here revealed that activation of TLR4 by Lipopolysaccharide (LPS) augments TNF- $\alpha$ production by human PBMCs previously exposed to L. braziliensis amastigotes, suggesting a crucial role for TLR4 in TNF- $\alpha$ induction and subsequently in controlling leishmanial infection [56]. Similar results were observed in a murine model of $L$. major immunization where LSA ability to reduce parasite load was statistically significantly increased when associated with TLR4a $[45,46]$. In view of the previous information together with the results presented here, TLR4 ligands should be also considerate in future studies as adjuvant for vaccines and immunotherapy in dogs.

In the present study, the effect of cytokine production of TLR3a was significantly less pronounced when compared with TLR4a and TLR7a. Interestingly, all dogs showed higher production of TNF- $\alpha$ after stimulation with TLR3a associated with LSA when compared with medium alone and with TLR3a. Recently, the essential role of TLR3a in inducing a Th1 response as adjuvant was demonstrated in a murine model of a Leishmania amazonensis vaccine based on total antigenic extract derived from promastigotes [57]. Therefore, TLR3a demonstrated its safety and ability to induce protection and its potential for being a promising candidate for Leishmania vaccines development. In contrast, other studies in which Leishmania RNA virus-1 (LRV1) was used to bind and stimulate endosomal TLR3 pathways in a murine model of Leishmania guayanensis infection confirmed that TLR3 is the dominant route of inflammatory pathology in this particular model $[58,59]$. Further research conducted on L. infantum infection in dogs is necessary to elucidate the potential use of TLR3a as vaccine adjuvant or immunotherapy in sick dogs.

TNF- $\alpha$ is a powerful proinflammatory cytokine that promotes together with IFN- $\gamma$ macrophage activation which is crucial for Leishmania parasite control both in dogs [60] and humans $[18,61]$. However, little has been investigated on the function of TNF- $\alpha$ and IL- 6 in dogs with clinical leishmaniosis. Moreover, less information is available related to dogs living in endemic areas of CanL and how these responses could affect the outcome of the disease in infected/exposed dogs. In the present study, a higher TNF- $\alpha$ response to LSA was observed when compared to medium alone in all dogs studied while this was not noted for IL-6, suggesting a more important role of TNF- $\alpha$ in inducing a proinflammatory response to Leishmania parasite. Accordingly, in previous studies, the use of TNF- $\alpha$ antagonists in subclinically infected people promoted the reactivation of infection by Leishmania spp. and the emergence of clinical signs of human leishmaniasis [62-64].

\section{Conclusion}

These findings indicate that TLR4a and TLR7a have a marked effect on cytokines production in ex vivo blood from apparently healthy dogs living in endemic areas of CanL. These data suggested an active role of TLRs on proinflammatory immune responses, which might be strongly involved in the process of disease resolution in dogs. In contrast, TLR3a seems to have a less pronounced effect on cytokine production in the present ex vivo model. Results presented here encourage further studies in order to evaluate the potential use of TLRa as vaccine adjuvant or as immunomodulatory therapy in Leishmania infection in dogs.

\section{Abreviations}

CanL: Canine leishmaniosis

$\mathrm{CO}_{2}$ : Carbon dioxide

CD4: Cluster of cuadruple differentiation

CMI: Cell-mediated immune

ConA: Concavalin A

DC: Dentritic cells

EDTA: Ethylenediaminetetraacetic acid

ELISA: Enzyme-linked immunosorbent assay

GLA-SE: Glucopyranosyl lipid adjuvant in stable emulsion

IFN- $\gamma$ : Interferon gamma

IL-12: Interleukin 12

IL-6: Interleukin 6

IMQ: Imiquimod-R837

iNos: Nitric oxide synthase

LPG: Lipophosphoglycan

LPS: Lipopolysaccharide

LRV1: Leishmania RNA virus-1

LSA: Leishmania soluble antigen

mL: $\quad$ Milliliters

MPLA: Monophosphoryl lipid A

NF- $\kappa$ B: Nuclear factor

kappa-light-chain-enhancer of activated B cells

NK: $\quad$ Natural killers

nm: Nanometers

NO: $\quad$ Nitric oxide

PBMCs: Peripheral blood mononuclear cells

PAMPs: Pathogen-associated molecular patterns

PRRs: Patter recognition receptors

ROS: Reactive oxygen species

SD: $\quad$ Standard deviation

Th1: $\quad$ Thelper 1

Th2: $\quad$ Thelper 2

TLRs: Toll-like receptors

TLR3: Toll-like receptors 3

TLR4: Toll-like receptors 4

TLR4: Toll-like receptors 7

TLRa: Toll-like receptor agonists 
TNF- $\alpha$ : Tumor necrosis factor alpha

VL: Visceral leishmaniosis

VLP: Virus-like particles

$\Phi: \quad$ Medium alone.

\section{Data Availability}

The data that support the findings of this study are available from the corresponding author, Laia Solano Gallego (LSG) upon reasonable request.

\section{Disclosure}

Dr. Laia Solano-Gallego held a Ramón y Cajal senior researcher contract awarded by the Ministerio de Ciencia e Innovación (Spain) and the European Social Fund.

\section{Conflicts of Interest}

The authors declare that they have no conflicts of interest.

\section{Authors' Contributions}

Laia Solano-Gallego designed the research study. Laia Solano-Gallego and Pamela Martínez-Orellana set up laboratory work. Laia Solano-Gallego coordinated the veterinary clinics enrolled. Laia Solano-Gallego and Paulina QuirolaAmores were involved in field studies. Pamela MartínezOrellana and Sara Montserrat-Sangrà performed whole blood assay and collection of supernatants. Pamela MartínezOrellana, Paulina Quirola-Amores, and Noemí González performed all serological and cytokine testing. Sara MontserratSangrà performed all RT-PCRs. Laia Solano-Gallego and Pamela Martínez-Orellana contributed with data analysis and interpretation. Laia Solano-Gallego and Pamela MartínezOrellana wrote and revised the manuscript. All authors read and approved the final version of the manuscript.

\section{Acknowledgments}

The authors want to thank all veterinarians and dog owners that contributed to this study. We are especially grateful to Oscar Cabezón (UAB) and Cati Lladó (Hospital Mon Veterinari, Mallorca) for collecting samples and Cèlia Cerezuela and Irina Casado Nuñez (UAB) who helped in laboratory work performance. This study was supported by Spanish Ministry grants, Ministerio de Economia y competitividad and FEDER, EU (AGL2012-32498 and AGL2015-68477).

\section{References}

[1] G. Baneth, A. F. Koutinas, L. Solano-Gallego, P. Bourdeau, and L. Ferrer, "Canine leishmaniosis-new concepts and insights on an expanding zoonosis: part one," Trends in Parasitology, vol. 24, no. 7, pp. 324-330, 2008.

[2] L. Solano-Gallego, A. Koutinas, G. Miró et al., "Directions for the diagnosis, clinical staging, treatment and prevention of canine leishmaniosis," Veterinary Parasitology, vol. 165, no. 1-2, pp. 1-18, 2009.
[3] E. Rossi, G. Bongiorno, E. Ciolli et al., "Seasonal phenology, host-blood feeding preferences and natural Leishmania infection of Phlebotomus perniciosus (Diptera, Psychodidae) in a high-endemic focus of canine leishmaniasis in Rome province, Italy," Acta Tropica, vol. 105, no. 2, pp. 158-165, 2008.

[4] L. Solano-Gallego, G. Mirá, A. Koutinas et al., "LeishVet guidelines for the practical management of canine leishmaniosis," Parasites \& Vectors, vol. 4, article 86, 2011.

[5] B. Alten, C. Maia, M. O. Afonso et al., "Seasonal Dynamics of Phlebotomine Sand Fly Species Proven Vectors of Mediterranean Leishmaniasis Caused by Leishmania infantum," PLOS Neglected Tropical Diseases, vol. 10, no. 2, p. e0004458, 2016.

[6] L. Solano-Gallego, L. Cardoso, M. G. Pennisi et al., "Diagnostic Challenges in the Era of Canine Leishmania infantum Vaccines," Trends in Parasitology, vol. 33, no. 9, pp. 706-717, 2017.

[7] A. Shokri, M. Fakhar, and S. H. Teshnizi, "Canine visceral leishmaniasis in Iran: A systematic review and meta-analysis," Acta Tropica, vol. 165, pp. 76-89, 2017.

[8] V. Sideris, G. Papadopoulou, E. Dotsika, and E. Karagouni, "Asymptomatic canine leishmaniasis in Greater Athens area, Greece," European Journal of Epidemiology, vol. 15, no. 3, pp. 271-276, 1999.

[9] V. Foglia Manzillo, T. Di Muccio, S. Cappiello et al., "Prospective study on the incidence and progression of clinical signs in naïve dogs naturally infected by Leishmania infantum," PLOS Neglected Tropical Diseases, vol. 7, no. 5, Article ID e2225, 2013.

[10] L. Solano-Gallego, J. Llull, G. Ramos et al., "The Ibizian hound presents a predominantly cellular immune response against natural Leishmania infection," Veterinary Parasitology, vol. 90, no. 1-2, pp. 37-45, 2000.

[11] E. Carrillo and J. Moreno, "Cytokine profiles in canine visceral leishmaniasis," Veterinary Immunology and Immunopathology, vol. 128, no. 1-3, pp. 67-70, 2009.

[12] F. Khadem and J. E. Uzonna, "Immunity to visceral leishmaniasis: Implications for immunotherapy," Future Microbiology, vol. 9, no. 7, pp. 901-915, 2014.

[13] L. Gradoni, "Canine Leishmania vaccines: Still a long way to go," Veterinary Parasitology, vol. 208, no. 1-2, pp. 94-100, 2015.

[14] C. L. Barbiéri, "Immunology of canine leishmaniasis," Parasite Immunology, vol. 28, no. 7, pp. 329-337, 2006.

[15] W. Kammoun-Rebai, N. Bahi-Jaber, I. Naouar et al., "Human cellular and humoral immune responses to Phlebotomus papatasi salivary gland antigens in endemic areas differing in prevalence of Leishmania major infection," PLOS Neglected Tropical Diseases, vol. 11, no. 10, 2017.

[16] D. Sacks and N. Noben-Trauth, "The immunology of susceptibility and resistance to Leishmania major in mice," Nature Reviews Immunology, vol. 2, no. 11, pp. 845-858, 2002.

[17] R. Zafra, J. R. Jaber, R. A. Pérez-Écija, A. Barragán, A. MartínezMoreno, and J. Pérez, "High iNOS expression in macrophages in canine leishmaniasis is associated with low intracellular parasite burden," Veterinary Immunology and Immunopathology, vol. 123, no. 3-4, pp. 353-359, 2008.

[18] S. J. Green, R. M. Crawford, J. T. Hockmeyer, M. S. Meltzer, and C. A. Nacy, "Leishmania major amastigotes initiate the $\mathrm{L}$-arginine-dependent killing mechanism in IFN- $\gamma$-stimulated macrophages by induction of tumor necrosis factor- $\alpha$," The Journal of Immunology, vol. 145, no. 12, pp. 4290-4297, 1990.

[19] P. Holzmuller, M. Hide, D. Sereno, and J.-L. Lemesre, "Leishmania infantum amastigotes resistant to nitric oxide cytotoxicity: impact on in vitro parasite developmental cycle and metabolic 
enzyme activities," Infection, Genetics and Evolution, vol. 6, no. 3, pp. 187-197, 2006.

[20] S. Hosein, D. P. Blake, and L. Solano-Gallego, "Insights on adaptive and innate immunity in canine leishmaniosis," Parasitology, vol. 144, no. 1, pp. 95-115, 2017.

[21] P. Tripathi, V. Singh, and S. Naik, "Immune response to Leishmania: Paradox rather than paradigm," FEMS Immunology \& Medical Microbiology, vol. 51, no. 2, pp. 229-242, 2007.

[22] L. Carrera, R. T. Gazzinelli, R. Badolato et al., "Leishmania promastigotes selectively inhibit interleukin 12 induction in bone marrow-derived macrophages from susceptible and resistant mice," The Journal of Experimental Medicine, vol. 183, no. 2, pp. 515-526, 1996.

[23] R. J. Quinnell, L. J. Kennedy, A. Barnes et al., "Susceptibility to visceral leishmaniasis in the domestic dog is associated with MHC class II polymorphism," Immunogenetics, vol. 55, no. 1, pp. 23-28, 2003.

[24] P. Martínez-Orellana, D. Marí-Martorell, S. Montserrat-Sangrà, L. Ordeix, G. Baneth, and L. Solano-Gallego, "Leishmania infantum-specific IFN- $\gamma$ production in stimulated blood from dogs with clinical leishmaniosis at diagnosis and during treatment," Veterinary Parasitology, vol. 248, pp. 39-47, 2017.

[25] L. Solano-Gallego, S. Montserrrat-Sangrà, L. Ordeix, and P. Martínez-Orellana, "Leishmania infantum-specific production of IFN- $\gamma$ and IL-10 in stimulated blood from dogs with clinical leishmaniosis," Parasites \& Vectors, vol. 9, no. 1, article no. 9, 2016.

[26] R. Medzhitov, “Toll-like receptors and innate immunity, Nature Reviews Immunology, vol. 1, no. 2, pp. 135-145, 2001.

[27] A. Cuscó, A. Sánchez, L. Altet, L. Ferrer, and O. Francino, “Nonsynonymous genetic variation in exonic regions of canine Tolllike receptors," Canine Genetics and Epidemiology, vol. 1, no. 1, p. 11, 2014.

[28] K. Takeda and S. Akira, "TLR signaling pathways," Seminars in Immunology, vol. 16, no. 1, pp. 3-9, 2004.

[29] H. Kumar, T. Kawai, and S. Akira, "Toll-like receptors and innate immunity," Biochemical and Biophysical Research Communications, vol. 388, no. 4, pp. 621-625, 2009.

[30] F. F. Tuon, V. S. Amato, H. A. Bacha, T. AlMusawi, M. I. Duarte, and V. A. Neto, “Toll-like receptors and leishmaniasis," Infection and Immunity, vol. 76, no. 3, pp. 866-872, 2008.

[31] P. Ganguli, S. Chowdhury, S. Chowdhury, and R. R. Sarkar, "Identification of Th1/Th2 regulatory switch to promote healing response during leishmaniasis: a computational approach," Eurasip Journal on Bioinformatics and Systems Biology, vol. 2015, no. 1, pp. 1-19, 2015.

[32] J.-F. Flandin, F. Chano, and A. Descoteaux, "RNA interference reveals a role for TLR2 and TLR3 in the recognition of Leishmania donovani promastigotes by interferon- $\gamma$-primed macrophages," European Journal of Immunology, vol. 36, no. 2, pp. 411-420, 2006.

[33] P. Kropf, M. A. Freudenberg, M. Modolell et al., "Toll-Like Receptor 4 Contributes to Efficient Control of Infection with the Protozoan Parasite Leishmania major," Infection and Immunity, vol. 72, no. 4, pp. 1920-1928, 2004.

[34] N. Craft, R. Birnbaum, N. Quanquin et al., "Topical Resiquimod Protects against Visceral Infection with Leishmania infantum chagasi in Mice," Clinical and Vaccine Immunology, vol. 21, no. 9, pp. 1314-1322, 2014.

[35] S. Gnjatic, N. B. Sawhney, and N. Bhardwaj, “Toll-like receptor agonists are they good adjuvants?" Cancer Journal, vol. 16, no. 4, pp. 382-391, 2010.
[36] F. Steinhagen, T. Kinjo, C. Bode, and D. M. Klinman, “TLRbased immune adjuvants," Vaccine, vol. 29, no. 17, pp. 3341-3355, 2011.

[37] F. L. Ribeiro-Gomes, M. C. A. Moniz-de-Souza, M. S. Alexandre-Moreira et al., "Neutrophils activate macrophages for intracellular killing of Leishmania major through recruitment of TLR4 by neutrophil elastase," The Journal of Immunology, vol. 179, no. 6, pp. 3988-3994, 2007.

[38] P. Martínez-Orellana, P. Quirola-Amores, S. Montserrat-Sangrà et al., "The inflammatory cytokine effect of Pam3CSK4 TLR2 agonist alone or in combination with Leishmania infantum antigen on ex-vivo whole blood from sick and resistant dogs," Parasites \& Vectors, vol. 10, no. 1, 2017.

[39] L. Ordeix, L. Solano-Gallego, D. Fondevila, L. Ferrer, and A. Fondati, "Papular dermatitis due to Leishmania spp. infection in dogs with parasite-specific cellular immune responses," Veterinary Dermatology, vol. 16, no. 3, pp. 187-191, 2005.

[40] L. Solano-Gallego, L. Di Filippo, L. Ordeix et al., "Early reduction of Leishmania infantum-specific antibodies and blood parasitemia during treatment in dogs with moderate or severe disease," Parasites \& Vectors, vol. 9, no. 1, article no. 235, 2016.

[41] F. Eskandari, G. A. Talesh, M. Parooie et al., "Immunoliposomes containing Soluble Leishmania Antigens (SLA) as a novel antigen delivery system in murine model of leishmaniasis," Experimental Parasitology emphasizes, vol. 146, pp. 78-86, 2014.

[42] D. Johnston and J.-C. Bystryn, "Topical imiquimod is a potent adjuvant to a weakly-immunogenic protein prototype vaccine," Vaccine, vol. 24, no. 11, pp. 1958-1965, 2006.

[43] R. D. Weeratna, S. R. Makinen, M. J. McCluskie, and H. L. Davis, "TLR agonists as vaccine adjuvants: comparison of CpG ODN and Resiquimod (R-848)," Vaccine, vol. 23, no. 45, pp. 52635270, 2005.

[44] W.-W. Zhang and G. Matlashewski, "Immunization with a Tolllike receptor 7 and/or 8 agonist vaccine adjuvant increases protective immunity against Leishmania major in BALB/c mice," Infection and Immunity, vol. 76, no. 8, pp. 3777-3783, 2008.

[45] T. Emami, S. M. Rezayat, A. Khamesipour et al., "The role of MPL and imiquimod adjuvants in enhancement of immune response and protection in $\mathrm{BALB} / \mathrm{c}$ mice immunized with soluble Leishmania antigen (SLA) encapsulated in nanoliposome," Artificial Cells, Nanomedicine and Biotechnology, pp. 1-10, 2018.

[46] M. Rostamian and H. M. Niknam, "Evaluation of the adjuvant effect of agonists of toll-like receptor 4 and 7/8 in a vaccine against leishmaniasis in BALB/c mice," Molecular Immunology, vol. 91, pp. 202-208, 2017.

[47] G. Khalili, F. Dobakhti, H. M. Niknam, V. Khaze, and F. Partovi, "Immunotherapy with Imiquimod Increases the Efficacy of Glucantime Therapy of Leishmania major Infection," Iranian Journal of Immunology, vol. 8, no. 1, pp. 45-51, 2011.

[48] D. Carter, C. B. Fox, T. A. Day et al., "A structure-function approach to optimizing TLR4 ligands for human vaccines," in Clinical \& Translational Immunology, vol. 5, p. e108, 11 edition, 2016.

[49] M. S. Duthie, L. Pereira, M. Favila et al., "A defined subunit vaccine that protects against vector-borne visceral leishmaniasis," NPJ Vaccines, vol. 2, no. 1, 2017.

[50] P. Cecílio, B. Pérez-Cabezas, L. Fernández et al., "Pre-clinical antigenicity studies of an innovative multivalent vaccine for human visceral leishmaniasis," PLOS Neglected Tropical Diseases, vol. 11, no. 11, p. e0005951, 2017. 
[51] S. Hosein, A. Rodríguez-Cortés, D. P. Blake et al., "Transcription of Toll-Like Receptors 2, 3, 4 and 9, FoxP3 and Th17 Cytokines in a Susceptible Experimental Model of Canine Leishmania infantum Infection," PLoS ONE, vol. 10, no. 10, p. e0140325, 2015.

[52] S. Antoniazi, H. P. Price, and P. Kropf, "Chemokine gene expression in Toll-like receptor-competent and -deficient mice infected with Leishmania major," Infection and Immunity, vol. 72, no. 9, pp. 5168-5174, 2004.

[53] S. Montserrat-Sangrà, L. Alborch, L. Ordeix, and L. SolanoGallego, "TLR-2 and TLR-4 transcriptions in unstimulated blood from dogs with leishmaniosis due to Leishmania infantum at the time of diagnosis and during follow-up treatment," Veterinary Parasitology, vol. 228, pp. 172-179, 2016.

[54] S. Montserrat-Sangrà, L. Ordeix, P. Martínez-Orellana, and L. Solano-Gallego, "Parasite Specific Antibody Levels, Interferon$\gamma$ and TLR2 and TLR4 Transcripts in Blood from Dogs with Different Clinical Stages of Leishmaniosis," Veterinary Sciences, vol. 5, no. 1, p. 31, 2018.

[55] A. Srivastava, N. Singh, M. Mishra et al., "Identification of TLR inducing Thl-responsive Leishmania donovani amastigotespecific antigens," Molecular and Cellular Biochemistry, vol. 359, no. 1-2, pp. 359-368, 2012.

[56] H. Galdino, R. Saar Gomes, J. C. dos Santos et al., "Leishmania (Viannia) braziliensis amastigotes induces the expression of TNF $\alpha$ and IL-10 by human peripheral blood mononuclear cells in vitro in a TLR4-dependent manner," Cytokine, vol. 88, pp. 184-192, 2016.

[57] M. V. Sanchez, R. J. Elicabe, M. S Genaro et al., "Total Leishmania antigens with Poly(I:C) induce Th1 protective response," Parasite Immunology, vol. 39, no. 11, 2017.

[58] R. O. Eren, M. Reverte, M. Rossi et al., "Mammalian Innate Immune Response to a Leishmania-Resident RNA Virus Increases Macrophage Survival to Promote Parasite Persistence," Cell Host \& Microbe, vol. 20, no. 3, pp. 318-328, 2016.

[59] M. Hartley, R. O. Eren, M. Rossi et al., "Leishmania guyanensis parasites block the activation of the inflammasome by inhibiting maturation of IL-1 $\beta$," Microbial Cell, vol. 5, no. 3, pp. 137-149, 2018.

[60] P. Holzmuller, M. Cavaleyra, J. Moreaux et al., "Lymphocytes of dogs immunised with purified excreted-secreted antigens of Leishmania infantum co-incubated with Leishmania infected macrophages produce IFN gamma resulting in nitric oxidemediated amastigote apoptosis," Veterinary Immunology and Immunopathology, vol. 106, no. 3-4, pp. 247-257, 2005.

[61] T. I. A. Roach, A. F. Kiderlen, and J. M. Blackwell, "Role of inorganic nitrogen oxides and tumor necrosis factor alpha in killing Leishmania donovani amastigotes in gamma interferonlipopolysaccharide-activated macrophages from Lsh(s) and Lsh(r) congenic mouse strains," Infection and Immunity, vol. 59, no. 11, pp. 3935-3944, 1991.

[62] M. B. Campos, L. V. Do Rêgo Lima, A. C. S. De Lima et al., “Tolllike receptors 2, 4, and 9 expressions over the entire clinical and immunopathological spectrum of American cutaneous leishmaniasis due to Leishmania (V.) braziliensis and Leishmania (L.) amazonensis," PLoS ONE, vol. 13, no. 3, 2018.

[63] A. L. C. Neumayr, G. Morizot, L. G. Visser et al., "Clinical aspects and management of cutaneous leishmaniasis in rheumatoid patients treated with TNF- $\alpha$ antagonists," Travel Medicine and Infectious Disease, vol. 11, no. 6, pp. 412-420, 2013.

[64] J. Marcoval and R. M. Penín, "Evolution of cutaneous leishmaniasis in the last 30 years in a tertiary hospital of the European
Mediterranean coast," International Journal of Dermatology, vol. 56, no. 7, pp. 750-753, 2017. 


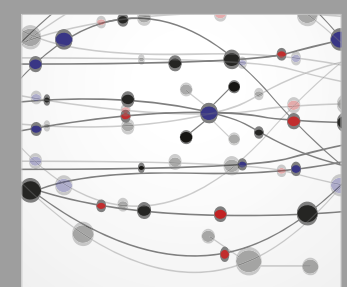

The Scientific World Journal
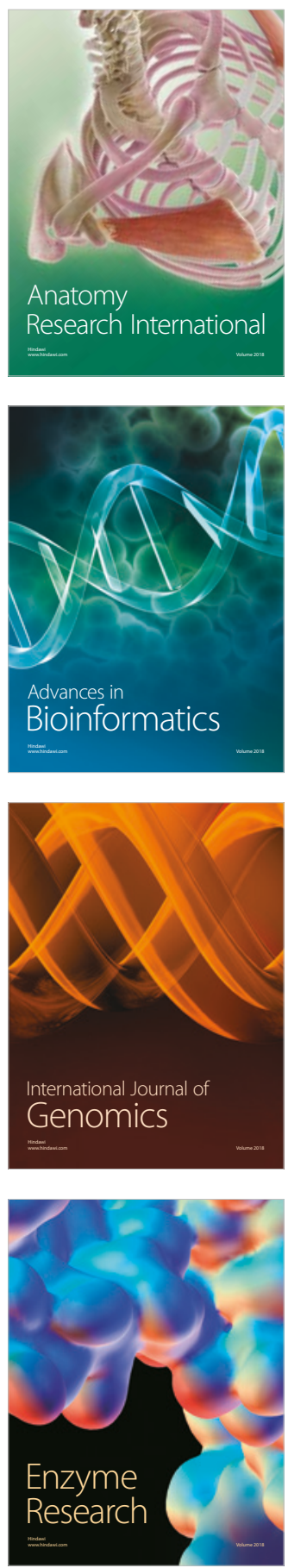
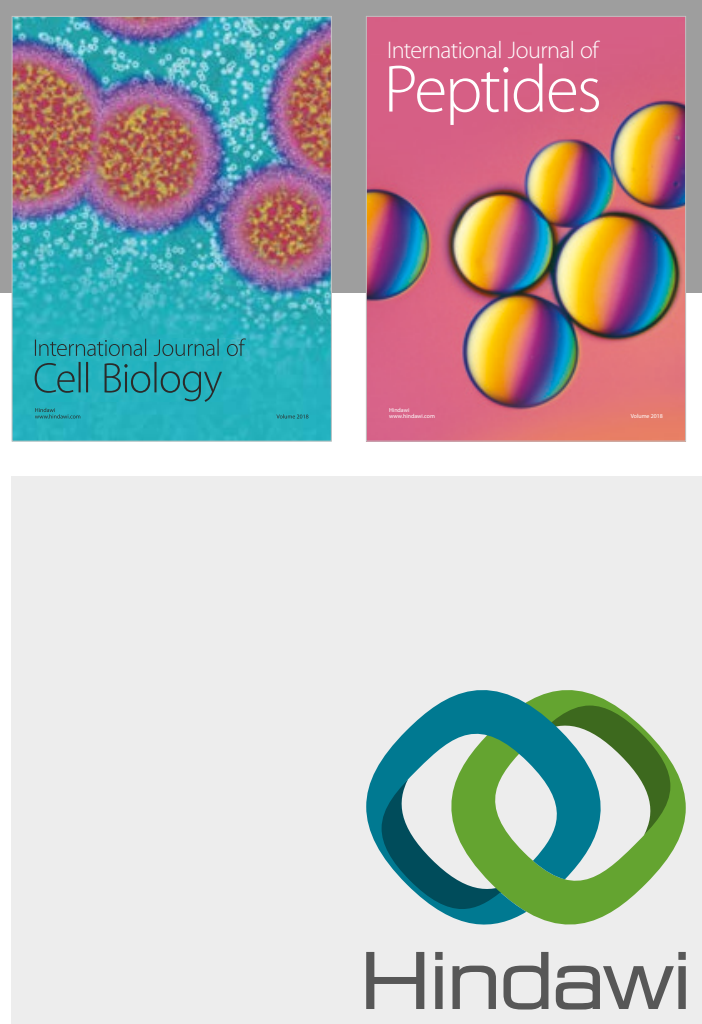

Submit your manuscripts at

www.hindawi.com
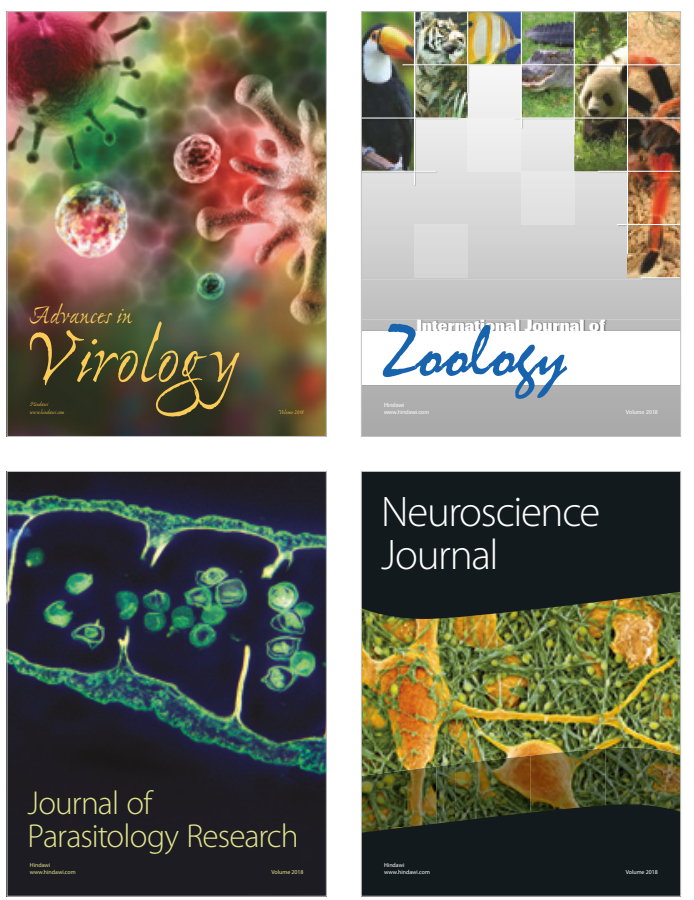
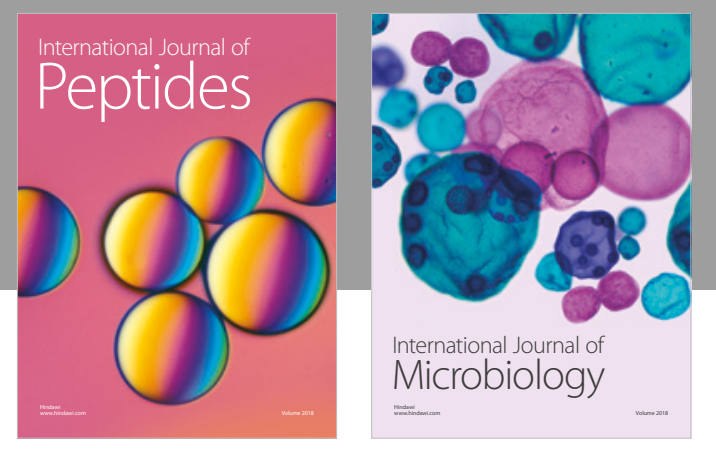

nternational Journal of Microbiology
Journal of
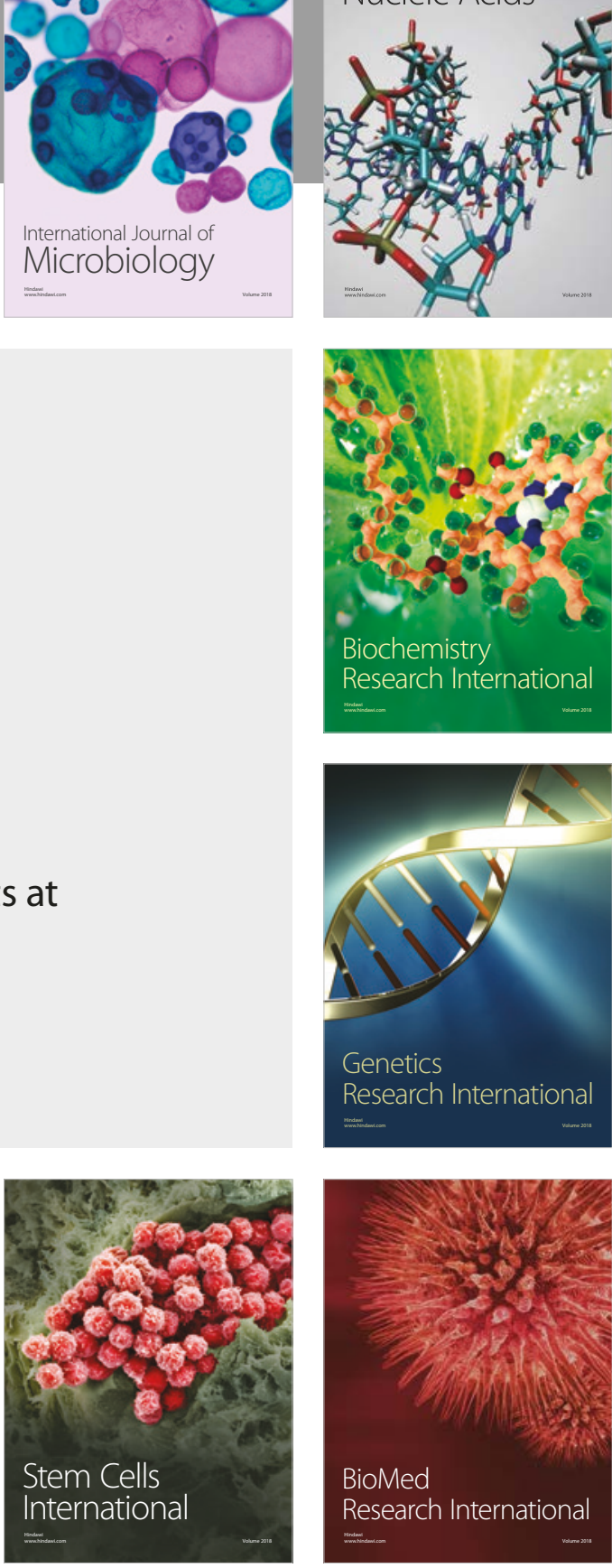
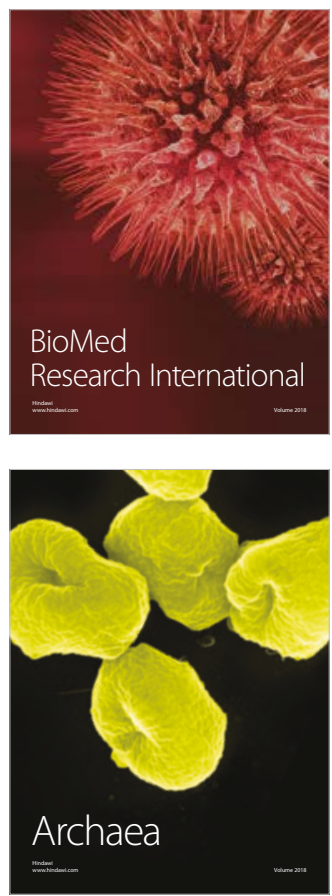